\title{
Number Crunching: How and When Will Numerical Models Be Used in the Clinical Setting?
}

W. Brent Edwards and Karen L. Troy

\section{Corresponding Author:}

Karen L. Troy

University of Illinois at Chicago,

Musculoskeletal Biomechanics Laboratories,

Department of Kinesiology and Nutrition,

Room 650, M/C 994,

1919 West Taylor Street ,

Chicago, IL 60612, USA

e-mail: klreed@uic.edu

The prevention of fractures remains the primary clinical goal for osteoporosis treatment.

Recently, improved understanding of the factors influencing the risk of osteoporotic fracture has made the application of personalized medicine possible in this arena. At the epidemiologic level, the FRAX (World Health Organization) tool provides individual fracture risk estimates [1]. However, even greater benefit could stem from patient-specific fracture risk assessment techniques independent of epidemiologic classification, as well as the objective identification of patients meeting requirements for a particular treatment (ie, exercise or pharmacologic 
intervention). Numerical modeling techniques will no doubt become an integral part of this medical practice. In the study of osteoporosis, numerical models are often adopted to predict the mechanical behavior of bone in vivo. In this context, numerical models have reached a level of accuracy necessary to extract clinically relevant information and help guide clinical decision making [2]. Although there are many types of numerical modeling techniques that can be used to examine the mechanical behavior of bone, we focus on the finite element method, as it is the most widely used and best developed technique for objects of complex geometry.

The finite element method is a computational technique that can be used to predict the mechanical behavior of a structure. In a process called “meshing," an object of complex geometry is divided into a system of small simplified geometries (eg, pyramids or cubes) called elements that are connected via discrete points called nodes. The inhomogeneity of the structure's material makeup can be captured by assigning different material properties to each individual element. These material properties are often based on information derived from imaging data, and there are many reports relating these types of data to mechanical behavior [35]. Boundary conditions such as loads and constraints are applied to the model. Equations relating the nodal forces and displacements to the applied force are assembled and solved simultaneously to obtain displacements, strains, and stresses throughout the entire geometry.

The use of finite element modeling in the field of osteoporosis research has grown substantially over the past two decades due to advances in computational power and imaging techniques. A primary use of the finite element method has been to estimate the fracture strength at specific anatomic locations, such as the distal radius [6], proximal femur [7], and vertebral body [8]. The fracture strength of whole bone is dependent on the bone's structural geometry, the distribution of mineral within the entire bone structure, the material properties of the bone, and 
the bone's mechanical loading environment. Unlike densitometry measures, finite element models capture this information, as well as the complex interaction between these factors, which leads to a substantial improvement in fracture strength prediction [8,9]. This aspect of the finite element method makes it an ideal integrative outcome measure to evaluate the success of a treatment or intervention [10], or to estimate the fracture risk of populations for whom epidemiologic norms have not been established, such as people living with spinal cord injury.

The accuracy in finite element predicted fracture strength is heavily dependent on the chosen failure criteria defining fracture threshold [11]. Despite the fact that bone material properties and strength are directionally dependent, or anisotropic, most subject-specific finite element models incorporate only isotropic material properties with uniaxial failure criteria. At least $90 \%$ of the anisotropy in trabecular bone material properties can be explained by a combination of structural density and alignment [12]. Unfortunately, apart from high-resolution peripheral quantitative CT devices, which are currently limited to peripheral skeletal sites such as the distal tibia and radius, clinical CT scanners lack the resolution needed to accurately quantify trabecular structural anisotropy. New promising methods are being developed to determine principal trabecular orientations directly from lower-resolution images [13]. These methods would allow for the implementation of multiaxial failure criteria, which in theory should further improve the accuracy in finite element predicted fracture strength.

Nearly all failure criteria currently being implemented within finite element routines are phenomenological in nature (ie,, they fail to address the underlying mechanisms of material failure). This restricts the applicability of finite element predicted fracture strength to stress/strain states similar to those used for model validation. Numerical models that incorporate mechanistic failure criteria, based on fracture mechanics [14] and cellular solid theory [15], can 
theoretically overcome this limitation. Of course some amount of error will always exist between modeled and empirical results simply due to the inherent variability of bone and limitations in the types of information that can be incorporated into models. The most clinically applicable fracture prediction techniques will likely be probabilistic in nature to account for these uncertainties $[16,17]$.

In addition to fracture strength prediction, finite element modeling has been used to simulate outcomes related to surgery and other interventions. These models typically simulate the bone adaptation process in response to alterations in the habitual mechanical loading environment. The first models in this area were used to guide the development of prosthetic hip stems that reduce stress shielding within the femur [18]. The more recent models are mechanistic, in that they incorporate a number of important mechanical and biological processes to answer questions related to exercise, overload, and disuse [19]. Although simulations of bone adaptation are still quite theoretical, advances in the field of mechanobiology will continue to strengthen and develop this field. Patient-specific finite element models may someday be used to simulate bone remodeling to design and evaluate customized orthopedic implants, or simulate the bone adaptation process in response to pharmacologic treatment with patient-specific loads imposed by activities of daily living.

Despite the numerous potentially important clinical applications for finite element modeling, several major barriers exist that prevent its incorporation into a clinical setting. First is the need for three-dimensional images, which are not otherwise routinely collected on patients. The additional expense and potential exposure to ionizing radiation means that patient-specific finite element models will never be routinely created for everyone. The challenge for the clinician will be in identifying those individuals who could benefit most from the information 
gained. Second, although a few groups have provided guidelines for the model verification and validation process to promote accuracy and increase peer acceptance of computational modeling techniques [2, 20], there is a potential need to develop standards relating to the acquisition and processing of images, meshing methods, material property assignment, boundary conditions, failure criteria, and so forth. Adoption of such standards by developers and investigators within the field would facilitate the comparison of results between researchers and, eventually, clinicians, increasing the utility of such models. A third major barrier is the degree of human expertise and hands-on time that is currently required to generate an accurate finite element model. In the context of bone, the process of finite element modeling has four steps: 1) segmentation of the biological structure from the imaging data, 2) generation of the finite element mesh, 3) assignment of material properties, and 4) application of constraints and loading conditions to the finite element model. The entirety of this process can be semiautomated at best. Until a more turn-key approach can be developed to generate such models, subject-specific finite element models are unlikely to be widely implemented outside the research setting.

In summary, the benefits of patient-specific finite element modeling as applied to osteoporosis research and treatment are clear. In their current state of development, finite element models represent the state of the art technique for fracture strength prediction. Clinicians may someday be able to use this information for better fracture risk assessment and to evaluate the success of a clinical treatment. The major challenges that prevent wider implementation of this technique include lack of standardized methods for generating and validating models, the expertise currently required to do so, and the additional cost and risks of acquiring threedimensional data. In this context, identifying which patients could most benefit from this type of 
personalized medicine will be important. A concerted effort should be made to address these current challenges.

\section{Disclosure}

Conflicts of interest: W.B. Edwards: has received grant support from Department of Defense (SC090010); K.L. Troy: is a consultant for Biomedical Forensics; has received grant support from National Institute on Disability and Rehabilitation Research (H133E070029); has received honoraria for an invited lecture from Rush University Medical Center and for an invited workshop from Dartmouth Medical School; and has received a travel award from the International Bone and Mineral Society.

\section{References}

1. Kanis JA, Oden A, Johansson H, et al.: FRAX and its applications to clinical practice. Bone 2009, 44: 734-743.

2. Viceconti M, Olsen S, Nolte LP, et al.: Extracting clinically relevant data from finite element simulations. Clinical Biomechanics 2005, 20: 451-454.

3. Snyder SM, Schneider E: Estimation of mechanical properties of cortical bone by computed tomography. Journal of Orthopaedic Research 1991, 9: 422-431.

4. Rho JY, Hobatho MC, Ashman, RB: Relations of mechanical properties to density and CT numbers in human bone. Medical Engineering \& Physics 1995, 17: 347-355.

5. Lotz JC, Gerhart TN, Hayes WC: Mechanical properties of trabecular bone from the proximal femur: A quantitative CT study. Journal of Computer Assisted Tomography 1990, 14: 107-114. 
6. Troy KL, Grabiner MD: Off-axis loads cause failure of the distal radius at lower magnitudes than axial loads: A finite element analysis. Journal of Biomechanics 2007, 40: 1670-1675.

7. Keyak JH, Rossi SA, Jones KA, et al.: Prediction of femoral fracture load using automated finite element modeling. Journal of Biomechanics 1998, 31: 125-133.

8. Crawford RP, Cann CE, Keaveny TM: Finite element models predict in vitro vertebral body compressive strength better than quantitative computed tomography. Bone 2003, 33: 744-750.

9. Cody DD, Gross GJ, Hou FJ, et al.: Femoral strength is better predicted by finite element models than QCT and DXA. Journal of Biomechanics 1999, 32: 1013-1020.

10. Keaveny TM, Hoffmann PF, Singh M, et al.: Femoral bone strength and its relation to cortical and trabecular changes after treatment with PTH, alendronate, and their combination as assessed by finite element analysis of quantitative CT scans. Journal of Bone and Mineral Research 2008, 23: 1974-1982.

11. Keyak JH, Rossi SA: Prediction of femoral fracture load using finite element models: An examination of stress- and strain-based failure theories. Journal of Biomechanics 2000, 33: 209214.

12. Homminga J, Mccreadie BR, Weinans $\mathrm{H}$, et al.: The dependence of the elastic properties of osteoporotic cancellous bone on volume fraction and fabric. Journal of Biomechanics 2003, 36: 1461-1467.

13. Tabor Z, Rokita E: Quantifying anisotropy of trabecular bone from gray-level images. Bone 2007, 40: 966-972.

14. Nalla RK, Kinney JH, Ritchie RO: Mechanistic fracture criteria for the failure of human cortical bone. Nature Materials 2003, 2: 164-168. 
15. Fenech CM, Keaveny TM: A cellular solid criterion for predicting the axial-shear failure properties of bovine trabecular bone. Journal of Biomechanical Engineering 1999, 121: 414-422. 16. Edwards WB, Taylor D, Rudolphi TJ, et al.: Effects of stride length and running mileage on a probabilistic stress fracture model. Medicine and Science in Sports and Exercise 2009, 41: 21772184.

17. Laz PJ, Stowe JQ, Baldwin MA, et al.: Incorporating uncertainty in mechanical properties for finite element-based evaluation of bone mechanics. Journal of Biomechanics 2007, 40: 28312836.

18. Huiskes R, Weinans H, Grootenboer HJ, et al.: Adaptive bone-remodeling theory applied to prosthetic-design analysis. Journal of Biomechanics 1987, 20: 1135-1150.

19. Hazelwood SJ, Castillo AB: Simulated effects of marathon training on bone density, remodeling, and microdamage accumulation of the femur. International Journal of Fatigue 2007, 29: 1057-1064.

20. Henninger HB, Reese SP, Anderson AE, et al.: Validation of computational models in biomechanics. Proceedings of the Institution of Mechanical Engineers. Part H, Journal of Engineering in Medicine 2009, 224: 801-812. 